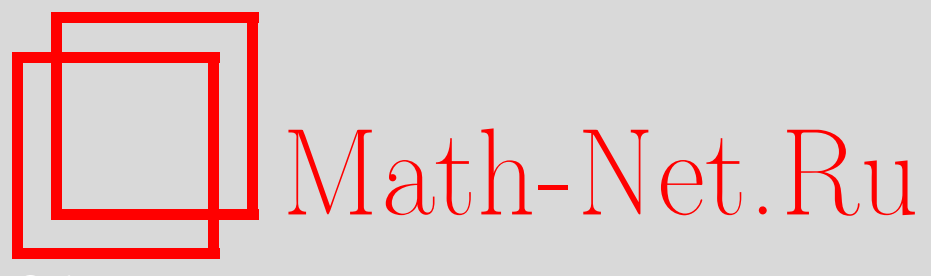

А. М. Селицкий, Пространство начальных данных 2-й краевой задачи для параболического дифференциальноразностного уравнения в липшицевых областях, Матем. заметки, 2013, том 94, выпуск 3, 477-480

DOI: https://doi.org/10.4213/mzm10307

Использование Общероссийского математического портала Math-Net.Ru подразумевает, что вы прочитали и согласны с пользовательским соглашением http: //www . mathnet.ru/rus/agreement

Параметры загрузки:

IP : 54.198 .55 .26

26 апреля 2023 г., 13:55:11

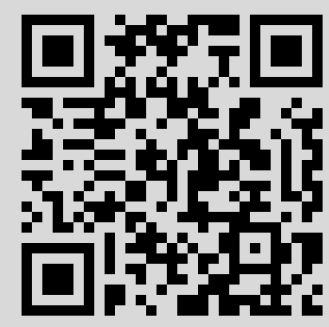




\section{Пространство начальных данных 2-й краевой задачи для параболического дифференциально-разностного уравнения в липшицевых областях}

\section{А. М. Селицкий}

1. Постановка задачи. Пусть $Q$ - ограниченная липшицева область в $\mathbb{R}^{n}$. Введем ограниченные разностные операторы $R_{i j}, R_{k}: L_{2}\left(\mathbb{R}^{n}\right) \rightarrow L_{2}\left(\mathbb{R}^{n}\right)$ по формулам

$$
\begin{aligned}
\left(R_{i j} u\right)(x) & =\sum_{h \in M} a_{i j, h} u(x+h), & i, j & =1, \ldots, n, \\
\left(R_{k} u\right)(x) & =\sum_{h \in M} a_{k, h} u(x+h), & k & =0,1, \ldots, n,
\end{aligned}
$$

$a_{i j, h}, a_{k, h}$ - комплексные числа, множество $M$ состоит из конечного набора векторов $h \in \mathbb{R}^{n}$ с целочисленными координатами.

Введем линейные ограниченные операторы: $I_{Q}: L_{2}(Q) \rightarrow L_{2}\left(\mathbb{R}^{n}\right)$ - оператор продолжения функции нулем вне $Q ; P_{Q}: L_{2}\left(\mathbb{R}^{n}\right) \rightarrow L_{2}(Q)$ - оператор сужения функции на $Q$, и операторы $R_{i j, Q}, R_{k, Q}: L_{2}(Q) \rightarrow L_{2}(Q)$, определенные равенствами $R_{i j, Q}=P_{Q} R_{i j} I_{Q}$, $R_{k, Q}=P_{Q} R_{k} I_{Q}$.

Будем рассматривать следующую задачу:

$$
\begin{gathered}
u_{t}-\sum_{i, j=1}^{n}\left(R_{i j, Q} u_{x_{j}}\right)_{x_{i}}+\sum_{k=1}^{n} R_{k, Q} u_{x_{k}}+R_{0, Q} u=f(x, t), \quad(x, t) \in Q_{T}, \\
\sum_{i j=1}^{n} R_{i j, Q} u_{x_{j}} \cos \left(\nu, x_{i}\right)=0, \quad(x, t) \in \Gamma_{T}, \\
\left.u\right|_{t=0}=\varphi(x), \quad x \in Q,
\end{gathered}
$$

где $f \in L_{2}\left(Q_{T}\right), \varphi \in L_{2}(Q), \nu$ - внешняя единичная нормаль (определенная почти всюду на $\partial Q), Q_{T}=Q \times(0, T), \Gamma_{T}=\partial Q \times(0, T)$.

Заметим, что вторая краевая задача для параболических дифференциально-разностных уравнений возникает в теории нелинейных оптических систем с двумерной обратной связью (см. [1] и приведенные там ссылки).

Чтобы сформулировать определение сильного решения задачи (1)-(3), рассмотрим полуторалинейную форму, определенную на пространстве Соболева $H^{1}(Q)$; через $(\cdot, \cdot)_{0},(\cdot, \cdot \cdot)_{1}$ будем обозначать скалярные произведения в $L_{2}(Q)$ и $H^{1}(Q)$, соответственно:

$$
a[v, w]=\sum_{i, j=1}^{n}\left(R_{i j, Q} v_{x_{j}}, w_{x_{i}}\right)_{0}+\sum_{k=1}^{n}\left(R_{k, Q} v_{x_{k}}, w\right)_{0}+\left(R_{0, Q} v, w\right)_{0} .
$$

Так как разностные операторы $R_{i j, Q}, R_{k, Q}$ ограничены в $L_{2}(Q)$, существует $c_{0}>0$ такое, что

$$
|a[v, w]| \leqslant c_{0}\|v\|_{1}\|w\|_{1}, \quad v, w \in H^{1}(Q) .
$$

Тогда существует линейный ограниченный оператор $A: H^{1}(Q) \rightarrow \widetilde{H}^{-1}(Q)\left(\right.$ где $\widetilde{H}^{-1}(Q)-$ сопряженное пространство к $H^{1}(Q)$ относительно скалярного произведения $\left.(\cdot, \cdot)_{0}\right)$, действующий по формуле

$$
\langle A v, \bar{w}\rangle=a[v, w], \quad v, w \in H^{1}(Q) .
$$

Работа выполнена при поддержке Российского фонда фундаментальных исследований (грант №№ 12-01-00524a, 13-01-00923).

DOI: $10.4213 / \mathrm{mzm} 10307$

(C) А. М. СЕлицкий, 2013 
ОПРедЕЛЕниЕ 1 . Оператор А будем называть сильно эллиптическим, если существуют числа $c_{1}>0$ и $c_{2} \geqslant 0$ такие, что

$$
\operatorname{Re} a[v, v] \geqslant c_{1}\|v\|_{1}^{2}-c_{2}\|v\|_{0}^{2}, \quad v \in H^{1}(Q) .
$$

Достаточные условия сильной эллиптичности оператора $A$ см., например, в лемме 2.2 в [2] (где второе слагаемое в (2.11) нужно читать $\left.k_{2}\|v\|_{L_{2}(Q)}^{2}\right)$.

В дальнейшем будем предполагать, что оператор $A$ сильно эллиптический. Заметим также, что вместо оператора $A$ можно рассматривать оператор $A+c_{2} I$, что соответствует замене функции $u$ в задаче (1)-(3) на $\tilde{u}=e^{c_{2} t} u$, а тогда в неравенстве (6) можно считать $c_{2}=0$.

В силу теоремы 2.1, гл. VI в [3], существует неограниченный оператор $\mathcal{A}: L_{2}(Q) \rightarrow L_{2}(Q)$ с областью определения $D(\mathcal{A}) \subset H^{1}(Q)$, действующий по формуле

$$
(\mathcal{A} v, w)_{0}=a[v, w], \quad v \in D(\mathcal{A}), \quad w \in H^{1}(Q),
$$

плотно и непрерывно.

Оператор $\mathcal{A}$ является сужением оператора $A$ на $D(\mathcal{A})$. Несложно проверить, что при предположении, что выполняется неравенство (6) с $c_{2}=0$, оператор $(-\mathcal{A})$ (а следовательно, и оператор $\mathcal{A}$ ) является замкнутым (см., например, теорему 4.5 гл. 1 в [4]), а тогда пространство $D(\mathcal{A})$ является гильбертовым с нормой графика. Введем гильбертово пространство $W(\mathcal{A})=\left\{w \in L_{2}([0, T] ; D(\mathcal{A})): w_{t} \in L_{2}\left(Q_{T}\right)\right\}$.

Из теоремы 3.2 в [2] следует, что задача (1)-(3) эквивалентна абстрактному операторному уравнению

$$
\frac{d u}{d t}+A u=f(t), \quad u(0)=\varphi,
$$

рассматриваемому в $L_{2}\left([0, T] ; L_{2}(Q)\right)=L_{2}\left(Q_{T}\right)$. Определим оператор $L: W(\mathcal{A}) \rightarrow L_{2}\left(Q_{T}\right)$ по формуле $L u(\cdot, t)=\mathcal{A} u(\cdot, t)$ при п.в. $t \in(0, T)$.

ОПРеДЕлЕниЕ 2. Силъным решением задачи (1)-(3) будем называть функцию $u \in$ $W(\mathcal{A})$, если она удовлетворяет уравнению

$$
\frac{d u}{d t}+L u=f \quad \text { при п.в. } t \in(0, T)
$$

и начальному условию

$$
\left.u\right|_{t=0}=\varphi .
$$

Конормальная производная в $(2)$ для функции из $H^{1}(Q)$ определяется из первой формулы Грина (ср. [5; п. 11.1]).

Напомним определение комплексной интерполяции, которая будет использоваться при описании пространства начальных данных. Пусть $X$ и $Y$-банаховы пространства и $Y \subset X$ плотно и непрерывно. Обозначим через $S$ полосу $S=\{z: 0<\operatorname{Re} z<1\}$. Через $\mathcal{H}(X, Y)$ обозначим пространство непрерывных ограниченных функций $F: \bar{S} \rightarrow Y$, аналитических в $S$, таких, что $F(i y) \in X$ непрерывна и ограничена, как $X$-значная функция от $y$, а $F(1+i y) \in Y$ непрерывна и ограничена, как $Y$-значная функция от $y$, с нормой

$$
\|F\|_{\mathcal{H}(X, Y)}=\max \left\{\sup _{-\infty<y<+\infty}\|F(i y)\|_{X}, \sup _{-\infty<y<+\infty}\|F(1+i y)\|_{Y}\right\} .
$$

ОПРеДЕЛЕНиЕ 3. Интерполяиионным пространством $[X, Y]_{\theta}$ называется образ пространства $\mathcal{H}(X, Y)$ при отображении $F \rightarrow F(\theta)$ :

$$
[X, Y]_{\theta}=\{f \in Y: \text { существует } F(z) \in \mathcal{H}(X, Y) \text { такая, что } F(\theta)=f\},
$$

с нормой

$$
\|f\|_{[X, Y]_{\theta}}=\inf _{F(\theta)=f}\|F\|_{\mathcal{H}(X, Y)} .
$$


Подробнее о комплексном методе интерполяции см. [6; гл. 1] в или [7].

Сформулируем теорему 4.2 из [2].

Tеорема 1. Пусть оператор А сильно эллиптический с $c_{2}=0$. Тогда для любых $f \in$ $L_{2}\left(Q_{T}\right)$ и $\varphi \in\left[L_{2}(Q), D(\mathcal{A})\right]_{1 / 2}$ задача (1)-(3) имеет единственное сильное решение, которое определяется по формуле

$$
u(x, t)=T_{t} \varphi(x)+\int_{0}^{t} T_{t-s} f(x, s) d s,
$$

где $\left\{T_{t}\right\} \quad(t \geqslant 0)$ - аналитическал полугруппа с генератором $(-\mathcal{A})$.

2. Пространство начальных данных. В теореме 1 принадлежность начальной функции пространству $\left[L_{2}(Q), D(\mathcal{A})\right]_{1 / 2}$ является необходимым и достаточным условием существования сильного решения (см. [8]).

Tеорема 2. Пустъ оператор А сильно эллиптический с $c_{2}=0$. Тогда

$$
\left[L_{2}(Q), D(\mathcal{A})\right]_{1 / 2}=H^{1}(Q) .
$$

ДоказАтельство. В силу теоремы 3.1 в $[9]\left[L_{2}(Q), D(\mathcal{A})\right]_{1 / 2}=D\left(\mathcal{A}^{1 / 2}\right)$. Поэтому для доказательства равенства (11) можно воспользоваться теоремой 3.2 из [10]. Для этого покажем, что оператор $A: H^{1+s}(Q) \rightarrow \widetilde{H}^{-1+s}(Q)$ является ограниченным при $|s|<1 / 2$.

Рассмотрим форму $a[v, w]$ на $H^{1+s}(Q) \times H^{1-s}(Q)$ :

$$
a[v, w]=\sum_{i, j=1}^{n}\left\langle R_{i j, Q} v_{x_{j}}, \bar{w}_{x_{i}}\right\rangle+\sum_{k=1}^{n}\left\langle R_{k, Q} v_{x_{k}}, \bar{w}\right\rangle+\left\langle R_{0, Q} v, \bar{w}\right\rangle .
$$

Если $v \in H^{1+s}(Q)$ и $w \in H^{1-s}(Q)$, то $v_{x_{j}} \in H^{s}(Q)$ и $w_{x_{i}} \in H^{-s}(Q)$. При $-1 / 2<s<0$ пространства $H^{s}$ совпадают с $\widetilde{H}^{s}$. Операторы $R_{i j, Q}, R_{k, Q}$ ограничены в $H^{s}(Q)$ при $|s|<1 / 2$, так как продолжение нулем - ограниченный оператор (при $s \geqslant 0$ см. теорему 3.33 [11], при $s<0$ см. следствие 3.6.4 в [5]). Таким образом, при каждом $|s|<1 / 2$ существует $C_{s}>0$, такое что

$$
|a[v, w]| \leqslant C_{s}\|v\|_{H^{1+s}(Q)}\|w\|_{H^{1-s}(Q)} .
$$

Тогда оператор $A$ определяется снова по формуле:

$$
\langle A v, \bar{w}\rangle=a[v, w], \quad v \in H^{1+s}(Q), \quad w \in H^{1-s}(Q),
$$

a $f=A v$ принадлежит $\widetilde{H}^{-1+s}(Q)$.

ЗАмечАниЕ. В [12] Като была сформулирована проблема для $m$-секториального оператора $A$ : "Верно ли, что $D\left(A^{1 / 2}\right)$ и $D\left(A^{* 1 / 2}\right)$ совпадают?" В [9] был построен пример оператора, не удовлетворяющего данному условию. Тогда вопрос остался, но для более узкого класса $m$-секториальных операторов - для операторов, заданных коэрцитивной формой. В [13] был построен контрпример и для такого класса операторов. Тем не менее, положительное решение этого вопроса для операторов, связанных с сильно эллиптическими системами дифференциальных уравнений с измеримыми ограниченными коэффициентами в липшицевых областях, получено в [14]. В статье [10] гипотеза Като была доказана для широкого класса операторов, заданных секториальной формой. Из теоремы 2 следует, что для операторов $\mathcal{A}$, рассмотренных в настоящей работе, справедливо равенство $D\left(\mathcal{A}^{1 / 2}\right)=D\left(\mathcal{A}^{* 1 / 2}\right)$ (оператор $\mathcal{A}^{*}$ определяется по полуторалинейной форме $\left.\overline{a[w, v]}\right)$, что является ответом на вопрос, поставленный в замечании 4.1 в [2]. В одномерном случае данный вопрос был решен положительно в [15]. Для дифференциально-разностных операторов с первым краевым условием данный вопрос решен положительно в [16].

Автор выражает благодарность А. Л. Скубачевскому за постановку задачи и постоянное внимание к работе, М. С. Аграновичу за длительное и плодотворное обсуждение вопросов, связанных с проблемой Т. Като, а также за прочтение рукописи и сделанные замечания, и А. А. Шкаликову за устранение неточности в заключительном замечании. 


\section{СПИСОК ЦИТИРОВАННОЙ ЛИТЕРАТУРЫ}

[1] A. L. Skubachevskii, Nonlinear Anal., 32:2 (1998), 261-278. [2] А. М. Селицкий, А. Л. Скубачевский, Тр. сем. им. И.Г. Петровского, 26, Изд-во Моск. ун-та, М., 2007, 324-347. [3] Т. Като, Теория возмущений линейных операторов, Мир, М., 1972. [4] А. Рazy, Semigroups of Linear Operators and Applications to Partial Differential Equations, Appl. Math. Sci., 44, Springer-Verlag, Berlin, 1983. [5] М. С. Агранович, Соболевские пространства, их обобщения и эллиптические задачи в областях с гладкой и липшицевой гранищей, МЦНМО, М., 2013. [6] Ж.-Л. Лионс, Э. Мадженес, Неоднородные граничные задачи и их приложения, Т. 1, Мир, М., 1971. [7] Х. Трибель, Теория интерполяции, функииональные пространства, дифференциальные операторы, Мир, М., 1980. [8] A. Ashyralyev, P. E. Sobolevskii, Well-Posedness of Parabolic Difference Equations, Oper. Theory Adv. Appl., 69, Birkhäuser Verlag, Basel, 1994. [9] J. L. Lions, J. Math. Soc. Japan, 14:2 (1962), 233-241. [10] М. С. Агранович, А.М. Селицкий, "Дробные степени операторов, отвечающих коэрцитивным задачам в липшицевых областях", Функи. анализ и его прил., 47:2 (2013), 2-17. [11] W. McLean, Strongly Elliptic Systems and Boundary Integral Equations, Cambridge Univ. Press, Cambridge, 2000. [12] T. Kato, J. Math. Soc. Japan, 13:3 (1961), 246-274. [13] A. McIntosh, Proc. Amer. Math. Soc., 32 (1972), 430-434. [14] A. Axelsson, S. Keith, A. McIntosh, J. London Math. Soc. (2), 74:1 (2006), 113-130. [15] А. М. Селицкий, Матем. заметки, 92:4 (2012), 636-640. [16] Р. В. Шамин, Матем. сб., 194:9 (2003), 141-156.

\section{А. М. Селицкий}

Поступило

Вычислительный центр

15.04 .2013

им. А. А. Дородницына РАН, г. Москва

Российский университет дружбы народов, г. Москва

E-mail: selitsky@mail.ru 